\title{
Global Attractivity and Periodic Character of Difference Equation of Order Four
}

\author{
M. A. Obaid, ${ }^{1}$ E. M. Elsayed, ${ }^{1,2}$ and M. M. El-Dessoky ${ }^{1,2}$ \\ ${ }^{1}$ Mathematics Department, Faculty of Science, King Abdulaziz University, P.O. Box 80203, \\ Jeddah 21589, Saudi Arabia \\ ${ }^{2}$ Department of Mathematics, Faculty of Science, Mansoura University, Mansoura 35516, Egypt
}

Correspondence should be addressed to M. M. El-Dessoky, dessokym@mans.edu.eg

Received 14 July 2012; Revised 2 September 2012; Accepted 17 September 2012

Academic Editor: Ibrahim Yalcinkaya

Copyright (C) 2012 M. A. Obaid et al. This is an open access article distributed under the Creative Commons Attribution License, which permits unrestricted use, distribution, and reproduction in any medium, provided the original work is properly cited.

We investigate the global convergence result, boundedness, and periodicity of solutions of the recursive sequence $x_{n+1}=a x_{n}+\left(\left(b x_{n-1}+c x_{n-2}+d x_{n-3}\right) /\left(\alpha x_{n-1}+\beta x_{n-2}+\gamma x_{n-3}\right)\right), n=0,1, \ldots$, where the parameters $a, b, c, d, \alpha, \beta$, and $\gamma$ are positive real numbers and the initial conditions $x_{-3}, x_{-2}, x_{-1}$, and $x_{0}$ are positive real numbers.

\section{Introduction}

Our goal in this paper is to investigate the global stability character, boundedness, and the periodicity of solutions of the recursive sequence

$$
x_{n+1}=a x_{n}+\frac{b x_{n-1}+c x_{n-2}+d x_{n-3}}{\alpha x_{n-1}+\beta x_{n-2}+\gamma x_{n-3}},
$$

where the parameters $a, b, c, d, \alpha, \beta$, and $\gamma$ are positive real numbers and the initial conditions $x_{-3}, x_{-2}, x_{-1}$, and $x_{0}$ are positive real numbers.

Recently there has been a lot of interest in studying the global attractivity, the boundedness character and the periodicity nature of nonlinear difference equations, see for example [1-15].

The study of the nonlinear rational difference equations of a higher order is quite challenging and rewarding, and the results about these equations offer prototypes towards the development of the basic theory of the global behavior of nonlinear difference equations of a big order, recently many researchers have investigated the behavior of the solution of 
difference equations-for example, in [3] Elabbasy et al. investigated the global stability, periodicity character and gave the solution of special case of the following recursive sequence:

$$
x_{n+1}=a x_{n}-\frac{b x_{n}}{c x_{n}-d x_{n-1}} .
$$

In [5] Elabbasy and Elsayed investigated the global stability character and boundedness of solutions of the recursive sequence

$$
x_{n+1}=\frac{a x_{n}^{p}+b \prod_{r=1}^{p} x_{n-r}}{c x_{n}^{p}+d \prod_{r=1}^{p} x_{n-r}} .
$$

Elsayed [11] investigated the global character of solutions of the nonlinear, fourth-order, rational difference equation

$$
x_{n+1}=a x_{n-2}+\frac{b x_{n} x_{n-2}}{c x_{n}+d x_{n-3}} .
$$

Saleh and Aloqeili [16] investigated the difference equation

$$
y_{n+1}=A+\frac{y_{n}}{y_{n-k}}
$$

Yang et al. [17] investigated the invariant intervals, the global attractivity of equilibrium points, and the asymptotic behavior of the solutions of the recursive sequence

$$
x_{n+1}=\frac{a x_{n-1}+b x_{n-2}}{c+d x_{n-1} x_{n-2}} .
$$

For some related work see [16-26].

Here, we recall some basic definitions and some theorems that we need in the sequel.

Let $I$ be some interval of real numbers and let

$$
F: I^{k+1} \longrightarrow I
$$

be a continuously differentiable function. Then for every set of initial conditions $x_{-k}, x_{-k+1}, \ldots, x_{0} \in I$, the difference equation

$$
x_{n+1}=F\left(x_{n}, x_{n-1}, \ldots, x_{n-k}\right), \quad n=0,1, \ldots
$$

has a unique solution $\left\{x_{n}\right\}_{n=-k}^{\infty}$. 
Definition 1.1 (Equilibrium Point). A point $\bar{x} \in I$ is called an equilibrium point of (1.8) if

$$
\bar{x}=F(\bar{x}, \bar{x}, \ldots, \bar{x}) .
$$

That is, $x_{n}=\bar{x}$ for $n \geq 0$ is a solution of (1.8), or equivalently, $\bar{x}$ is a fixed point of $f$.

Definition 1.2 (Periodicity). A sequence $\left\{x_{n}\right\}_{n=-k}^{\infty}$ is said to be periodic with period $p$ if $x_{n+p}=$ $x_{n}$ for all $n \geq-k$.

Definition 1.3 (Stability). (i) The equilibrium point $\bar{x}$ of (1.8) is locally stable if for every $\epsilon>$ 0 , there exists $\delta>0$ such that for all $x_{-k}, x_{-k+1}, \ldots, x_{-1}, x_{0} \in I$ with

$$
\left|x_{-k}-\bar{x}\right|+\left|x_{-k+1}-\bar{x}\right|+\cdots+\left|x_{0}-\bar{x}\right|<\delta
$$

we have

$$
\left|x_{n}-\bar{x}\right|<\epsilon \quad \forall n \geq-k
$$

(ii) The equilibrium point $\bar{x}$ of (1.8) is locally asymptotically stable if $\bar{x}$ is locally stable solution of (1.8) and there exists $\gamma>0$, such that for all $x_{-k}, x_{-k+1}, \ldots, x_{-1}, x_{0} \in I$ with

$$
\left|x_{-k}-\bar{x}\right|+\left|x_{-k+1}-\bar{x}\right|+\cdots+\left|x_{0}-\bar{x}\right|<\gamma,
$$

we have

$$
\lim _{n \rightarrow \infty} x_{n}=\bar{x}
$$

(iii) The equilibrium point $\bar{x}$ of (1.8) is a global attractor if for all $x_{-k}$, $x_{-k+1}, \ldots, x_{-1}, x_{0} \in I$, we have

$$
\lim _{n \rightarrow \infty} x_{n}=\bar{x}
$$

(iv) The equilibrium point $\bar{x}$ of (1.8) is globally asymptotically stable if $\bar{x}$ is locally stable, and $\bar{x}$ is also a global attractor of (1.8).

(v) The equilibrium point $\bar{x}$ of (1.8) is unstable if $\bar{x}$ is not locally stable. equation

The linearized equation of (1.8) about the equilibrium $\bar{x}$ is the linear difference

$$
y_{n+1}=\sum_{i=0}^{k} \frac{\partial F(\bar{x}, \bar{x}, \ldots, \bar{x})}{\partial x_{n-i}} y_{n-i}
$$

Theorem A (see [18]). Assume that $p_{i} \in R, i=1,2, \ldots, k$ and $k \in\{0,1,2, \ldots\}$. Then

$$
\sum_{i=1}^{k}\left|p_{i}\right|<1
$$


is a sufficient condition for the asymptotic stability of the difference equation

$$
x_{n+k}+p_{1} x_{n+k-1}+\cdots+p_{k} x_{n}=0 \quad n=0,1, \ldots
$$

Theorem B (see [19]). Let $g:[a, b]^{k+1} \rightarrow[a, b]$ be a continuous function, where $k$ is a positive integer, and where $[a, b]$ is an interval of real numbers. Consider the difference equation

$$
x_{n+1}=g\left(x_{n}, x_{n-1}, \ldots, x_{n-k}\right), \quad n=0,1, \ldots
$$

Suppose that $g$ satisfies the following conditions.

(1) For each integer $i$ with $1 \leq i \leq k+1$; the function $g\left(z_{1}, z_{2}, \ldots, z_{k+1}\right)$ is weakly monotonic in $z_{i}$ for fixed $z_{1}, z_{2}, \ldots, z_{i-1}, z_{i+1}, \ldots, z_{k+1}$.

(2) If $(m, M)$ is a solution of thmhje system

$$
m=g\left(m_{1}, m_{2}, \ldots, m_{k+1}\right), \quad M=g\left(M_{1}, M_{2}, \ldots, M_{k+1}\right),
$$

then $m=M$, where for each $i=1,2, \ldots, k+1$, we set

$$
\begin{aligned}
m_{i} & = \begin{cases}m & \text { if } g \text { is nondecreasing in } z_{i}, \\
M & \text { if } g \text { is nonincreasing in } z_{i},\end{cases} \\
M_{i} & = \begin{cases}M & \text { if } g \text { is nondecreasing in } z_{i}, \\
m & \text { if } g \text { is nonincreasing in } z_{i} .\end{cases}
\end{aligned}
$$

Then there exists exactly one equilibrium $\bar{x}$ of (1.18), and every solution of (1.18) converges to $\bar{x}$.

The paper proceeds as follows. In Section 2, we show that when

$$
(\alpha+\beta+\gamma)(b+c+d)>\max \left\{\begin{array}{c}
|2 \alpha(c+d)-2 b(\beta+\gamma)|,|2 \gamma(b+c)-2 d(\alpha+\beta)|, \\
|2 \beta(b+d)-2 c(\alpha+\gamma)|
\end{array}\right\}
$$

then the equilibrium point of (1.1) is locally asymptotically stable. In Section 3 we prove that the solution is bounded and persists when $a<1$ and the solution of (1.1) is unbounded if $a>1$. In Section 4 we prove that there exists a period two solution of (1.1). In Section 5 we prove that the equilibrium point of (1.1) is global attractor. Finally we give a numerical examples of some special cases of (1.1) and draw it by using Matlab.

\section{Local Stability of the Equilibrium Point of (1.1)}

This section deals with study of the local stability character of the equilibrium point of (1.1).

Equation (1.1) has equilibrium point and is given by

$$
\bar{x}=a \bar{x}+\frac{b+c+d}{\alpha+\beta+\gamma} .
$$


If $a<1$, then the only positive equilibrium point of (1.1) is given by

$$
\bar{x}=\frac{b+c+d}{(\alpha+\beta+\gamma)(1-a)} .
$$

Let $f:(0, \infty)^{4} \rightarrow(0, \infty)$ be a continuously differentiable function defined by

$$
f(u, v, w, t)=a u+\frac{b v+c w+d t}{\alpha v+\beta w+\gamma t}
$$

Therefore, it follows that

$$
\begin{gathered}
\frac{\partial f(u, v, w, t)}{\partial u}=a \\
\frac{\partial f(u, v, w, t)}{\partial v}=\frac{(b \beta-c \alpha) w+(b \gamma-d \alpha) t}{(\alpha v+\beta w+\gamma t)^{2}} \\
\frac{\partial f(u, v, w, t)}{\partial w}=\frac{-(b \beta-c \alpha) v+(c \gamma-d \beta) t}{(\alpha v+\beta w+\gamma t)^{2}} \\
\frac{\partial f(u, v, w, t)}{\partial t}=\frac{-(b \gamma-d \alpha) v-(c \gamma-d \beta) w}{(\alpha v+\beta w+\gamma t)^{2}} .
\end{gathered}
$$

Then we see that

$$
\begin{gathered}
\frac{\partial f(\bar{x}, \bar{x}, \bar{x}, \bar{x})}{\partial u}=a=-a_{3}, \\
\frac{\partial f(\bar{x}, \bar{x}, \bar{x}, \bar{x})}{\partial v}=\frac{(b \beta-c \alpha)+(b \beta-d \alpha)}{(\alpha+\beta+\gamma)^{2} \bar{x}}=\frac{[(b \beta-c \alpha)+(b \gamma-d \alpha)](1-a)}{(\alpha+\beta+\gamma)(b+c+d)}=-a_{2} \\
\frac{\partial f(\bar{x}, \bar{x}, \bar{x}, \bar{x})}{\partial w}=\frac{-(b \beta-c \alpha)+(c \gamma-d \beta)}{(\alpha+\beta+\gamma)^{2} \bar{x}}=\frac{[-(b \beta-c \alpha)+(c \gamma-d \beta)](1-a)}{(\alpha+\beta+\gamma)(b+c+d)}=-a_{1}, \\
\frac{\partial f(\bar{x}, \bar{x}, \bar{x}, \bar{x})}{\partial t}=\frac{-(b \gamma-d \alpha)-(c \gamma-d \beta)}{(\alpha+\beta+\gamma)^{2} \bar{x}}=\frac{[-(b \gamma-d \alpha)-(c \gamma-d \beta)](1-a)}{(\alpha+\beta+\gamma)(b+c+d)}=-a_{0} .
\end{gathered}
$$

Then the linearized equation of (1.1) about $\bar{x}$ is

$$
y_{n+1}+a_{3} y_{n}+a_{2} y_{n-1}+a_{1} y_{n-2}+a_{0} y_{n-3}=0
$$

whose characteristic equation is

$$
\lambda^{4}+a_{3} \lambda^{3}+a_{2} \lambda^{2}+a_{1} \lambda+a_{0}=0
$$


Theorem 2.1. Assume that

$$
(\alpha+\beta+\gamma)(b+c+d)>\max \left\{\begin{array}{l}
|2 \alpha(c+d)-2 b(\beta+\gamma)|, \\
|2 \gamma(b+c)-2 d(\alpha+\beta)| \\
|2 \beta(b+d)-2 c(\alpha+\gamma)|
\end{array}\right\}
$$

Then the positive equilibrium point of (1.1) is locally asymptotically stable.

Proof. It is follows by Theorem A that (2.6) is asymptotically stable if all roots of (2.7) lie in the open $\operatorname{disc}|\lambda|<1$, that is, if

$$
\begin{gathered}
\left|a_{3}\right|+\left|a_{2}\right|+\left|a_{1}\right|+\left|a_{0}\right|<1 \\
|a|+\left|\frac{[(b \beta-c \alpha)+(b \gamma-d \alpha)](1-a)}{(\alpha+\beta+\gamma)(b+c+d)}\right|+\left|\frac{[-(b \beta-c \alpha)+(c \gamma-d \beta)](1-a)}{(\alpha+\beta+\gamma)(b+c+d)}\right| \\
+\left|\frac{[-(b \gamma-d \alpha)-(c \gamma-d \beta)](1-a)}{(\alpha+\beta+\gamma)(b+c+d)}\right|<1
\end{gathered}
$$

and so

$$
\begin{aligned}
& |[(b \beta-c \alpha)+(b \gamma-d \alpha)](1-a)|+|[-(b \beta-c \alpha)+(c \gamma-d \beta)](1-a)| \\
& \quad+|[-(b \gamma-d \alpha)-(c \gamma-d \beta)](1-a)|<[(\alpha+\beta+\gamma)(b+c+d)](1-a)
\end{aligned}
$$

Dividing the denominator and numerator by $(1-a)$ gives

$$
\begin{aligned}
& |(b \beta-c \alpha)+(b \gamma-d \alpha)|+|-(b \beta-c \alpha)+(c \gamma-d \beta)| \\
& +|-(b \gamma-d \alpha)-(c \gamma-d \beta)|<(\alpha+\beta+\gamma)(b+c+d)
\end{aligned}
$$

Suppose that

$$
\begin{aligned}
& B_{1}=(b \beta-c \alpha)+(b \gamma-d \alpha), \\
& B_{2}=-(b \beta-c \alpha)+(c \gamma-d \beta), \\
& B_{3}=-(b \gamma-d \alpha)-(c \gamma-d \beta) .
\end{aligned}
$$

We consider the following cases.

(1) $B_{1}>0, B_{2}>0$, and $B_{3}>0$. In this case we see from (2.11) that

$$
\begin{gathered}
(b \beta-c \alpha)+(b \gamma-d \alpha)-(b \beta-c \alpha)+(c \gamma-d \beta)-(b \gamma-d \alpha) \\
-(c \gamma-d \beta)<(\alpha+\beta+\gamma)(b+c+d)
\end{gathered}
$$


if and only if

$$
(\alpha+\beta+\gamma)(b+c+d)>0
$$

which is always true.

(2) $B_{1}>0, B_{2}>0$, and $B_{3}<0$. It follows from (2.11) that

$$
\begin{aligned}
& (b \beta-c \alpha)+(b \gamma-d \alpha)-(b \beta-c \alpha)+(c \gamma-d \beta)+(b \gamma-d \alpha)+(c \gamma-d \beta) \\
& <(\alpha+\beta+\gamma)(b+c+d)
\end{aligned}
$$

if and only if

$$
2 \gamma(b+c)-2 d(\alpha+\beta)<(\alpha+\beta+\gamma)(b+c+d),
$$

which is satisfied by (2.8).

(3) $B_{1}>0, B_{2}<0$, and $B_{3}>0$. We see from (2.11) that

$$
\begin{gathered}
(b \beta-c \alpha)+(b \gamma-d \alpha)+(b \beta-c \alpha)-(c \gamma-d \beta)-(b \gamma-d \alpha) \\
-(c \gamma-d \beta)<(\alpha+\beta+\gamma)(b+c+d)
\end{gathered}
$$

if and only if

$$
2 \beta(b+d)-2 c(\alpha+\gamma)<(\alpha+\beta+\gamma)(b+c+d),
$$

which is satisfied by (2.8).

(4) $B_{1}>0, B_{2}<0$, and $B_{3}<0$. It follows from (2.11) that

$$
\begin{aligned}
& (b \beta-c \alpha)+(b \gamma-d \alpha)+(b \beta-c \alpha)-(c \gamma-d \beta)+(b \gamma-d \alpha)+(c \gamma-d \beta) \\
& <(\alpha+\beta+\gamma)(b+c+d)
\end{aligned}
$$

if and only if

$$
2 b(\beta+\gamma)-2 \alpha(c+d)<(\alpha+\beta+\gamma)(b+c+d),
$$

which is satisfied by (2.8).

(5) $B_{1}<0, B_{2}>0$, and $B_{3}>0$. We see from (2.11) that

$$
\begin{aligned}
& -(b \beta-c \alpha)-(b \gamma-d \alpha)-(b \beta-c \alpha)+(c \gamma-d \beta)-(b \gamma-d \alpha)-(c \gamma-d \beta) \\
& \quad<(\alpha+\beta+\gamma)(b+c+d)
\end{aligned}
$$


if and only if

$$
2 \alpha(c+d)-2 b(\beta+\gamma)<(\alpha+\beta+\gamma)(b+c+d),
$$

which is satisfied by (2.8).

(6) $B_{1}<0, B_{2}>0$, and $B_{3}<0$. It follows from (2.11) that

$$
\begin{gathered}
-(b \beta-c \alpha)-(b \gamma-d \alpha)-(b \beta-c \alpha)+(c \gamma-d \beta)+(b \gamma-d \alpha) \\
+(c \gamma-d \beta)<(\alpha+\beta+\gamma)(b+c+d)
\end{gathered}
$$

if and only if

$$
2 c(\alpha+\gamma)-2 \beta(b+d)<(\alpha+\beta+\gamma)(b+c+d),
$$

which is satisfied by (2.8).

(7) $B_{1}<0, B_{2}<0$, and $B_{3}>0$. We see from (2.11) that

$$
\begin{gathered}
-(b \beta-c \alpha)-(b \gamma-d \alpha)+(b \beta-c \alpha)-(c \gamma-d \beta)-(b \gamma-d \alpha)-(c \gamma-d \beta) \\
<(\alpha+\beta+\gamma)(b+c+d)
\end{gathered}
$$

if and only if

$$
2 d(\alpha+\beta)-2 \gamma(b+c)<(\alpha+\beta+\gamma)(b+c+d),
$$

which is satisfied by (2.8).

(8) $B_{1}<0, B_{2}<0$, and $B_{3}<0$. It follows from (2.11) that

$$
\begin{aligned}
- & (b \beta-c \alpha)-(b \gamma-d \alpha)+(b \beta-c \alpha)-(c \gamma-d \beta)+(b \gamma-d \alpha)+(c \gamma-d \beta) \\
& <(\alpha+\beta+\gamma)(b+c+d)
\end{aligned}
$$

if and only if

$$
(\alpha+\beta+\gamma)(b+c+d)>0
$$

which is always true. The proof is complete.

\section{Boundedness of Solutions of (1.1)}

Here we study the boundedness nature and persistence of solutions of (1.1). 
Theorem 3.1. Every solution of (1.1) is bounded and persists if $a<1$.

Proof. Let $\left\{x_{n}\right\}_{n=-3}^{\infty}$ be a solution of (1.1). It follows from (1.1) that

$$
\begin{aligned}
x_{n+1}= & a x_{n}+\frac{b x_{n-1}+c x_{n-2}+d x_{n-3}}{\alpha x_{n-1}+\beta x_{n-2}+\gamma x_{n-3}}=a x_{n}+\frac{b x_{n-1}}{\alpha x_{n-1}+\beta x_{n-2}+\gamma x_{n-3}} \\
& +\frac{c x_{n-2}}{\alpha x_{n-1}+\beta x_{n-2}+\gamma x_{n-3}}+\frac{d x_{n-3}}{\alpha x_{n-1}+\beta x_{n-2}+\gamma x_{n-3}} .
\end{aligned}
$$

Then

$$
x_{n+1} \leq a x_{n}+\frac{b x_{n-1}}{\alpha x_{n-1}}+\frac{c x_{n-2}}{\beta x_{n-2}}+\frac{d x_{n-3}}{\gamma x_{n-3}}=a x_{n}+\frac{b}{\alpha}+\frac{c}{\beta}+\frac{d}{r} \quad \forall n \geq 1 .
$$

By using a comparison, we see that

$$
\limsup _{n \rightarrow \infty} x_{n} \leq \frac{b \beta \gamma+c \alpha \gamma+d \alpha \beta}{\alpha \beta \gamma(1-a)}=M
$$

Thus the solution is bounded.

Now we wish to show that there exists $m>0$ such that

$$
x_{n} \geq m \quad \forall n \geq 1
$$

The transformation

$$
x_{n}=\frac{1}{y_{n}}
$$

will reduce (1.1) to the equivalent form

$$
\frac{1}{y_{n+1}}=\frac{a\left(\alpha y_{n-2} y_{n-3}+\beta y_{n-1} y_{n-3}+\gamma y_{n-1} y_{n-2}\right)+y_{n}\left(b y_{n-2} y_{n-3}+c y_{n-1} y_{n-3}+d y_{n-1} y_{n-2}\right)}{y_{n}\left(\alpha y_{n-2} y_{n-3}+\beta y_{n-1} y_{n-3}+\gamma y_{n-1} y_{n-2}\right)}
$$

or

$$
y_{n+1}=\frac{y_{n}\left(\alpha y_{n-2} y_{n-3}+\beta y_{n-1} y_{n-3}+\gamma y_{n-1} y_{n-2}\right)}{a\left(\alpha y_{n-2} y_{n-3}+\beta y_{n-1} y_{n-3}+\gamma y_{n-1} y_{n-2}\right)+y_{n}\left(b y_{n-2} y_{n-3}+c y_{n-1} y_{n-3}+d y_{n-1} y_{n-2}\right)}
$$


It follows that

$$
\begin{aligned}
y_{n+1} \leq & \frac{y_{n}\left(\alpha y_{n-2} y_{n-3}+\beta y_{n-1} y_{n-3}+r y_{n-1} y_{n-2}\right)}{y_{n}\left(b y_{n-2} y_{n-3}+c y_{n-1} y_{n-3}+d y_{n-1} y_{n-2}\right)} \\
= & \frac{\alpha y_{n-2} y_{n-3}}{b y_{n-2} y_{n-3}+c y_{n-1} y_{n-3}+d y_{n-1} y_{n-2}}+\frac{\beta y_{n-1} y_{n-3}}{b y_{n-2} y_{n-3}+c y_{n-1} y_{n-3}+d y_{n-1} y_{n-2}} \\
& +\frac{r y_{n-1} y_{n-2}}{b y_{n-2} y_{n-3}+c y_{n-1} y_{n-3}+d y_{n-1} y_{n-2}} \\
\leq & \frac{\alpha y_{n-2} y_{n-3}}{b y_{n-2} y_{n-3}}+\frac{\beta y_{n-1} y_{n-3}}{c y_{n-1} y_{n-3}}+\frac{r y_{n-1} y_{n-2}}{d y_{n-1} y_{n-2}}=\frac{\alpha}{b}+\frac{\beta}{c}+\frac{\gamma}{d} \\
= & \frac{\alpha c d+\beta b d+\gamma b c}{b c d}=H \quad \forall n \geq 1 .
\end{aligned}
$$

Thus we obtain

$$
x_{n}=\frac{1}{y_{n}} \geq \frac{1}{H}=\frac{b c d}{\alpha c d+\beta b d+\gamma b c}=m \quad \forall n \geq 1 .
$$

From (3.3) and (3.9), we see that

$$
m \leq x_{n} \leq M \quad \forall n \geq 1
$$

Therefore, every solution of (1.1) is bounded and persists.

Theorem 3.2. Every solution of (1.1) is unbounded if $a>1$.

Proof. Let $\left\{x_{n}\right\}_{n=-3}^{\infty}$ be a solution of (1.1). Then from (1.1) we see that

$$
x_{n+1}=a x_{n}+\frac{b x_{n-1}+c x_{n-2}+d x_{n-3}}{\alpha x_{n-1}+\beta x_{n-2}+\gamma x_{n-3}}>a x_{n} \quad \forall n \geq 1 .
$$

We see that the right-hand side can be written as follows:

$$
y_{n+1}=a y_{n} \Longrightarrow y_{n}=a^{n} y_{0}
$$

and this equation is unstable because $a>1$, and $\lim _{n \rightarrow \infty} y_{n}=\infty$. Then by using ratio test $\left\{x_{n}\right\}_{n=-3}^{\infty}$ is unbounded from above.

\section{Existence of Periodic Solutions}

In this section we study the existence of periodic solutions of (1.1). The following theorem states the necessary and sufficient conditions that this equation has periodic solutions of prime period two. 
Theorem 4.1. Equation (1.1) has positive prime period two solutions if and only if

(i) $(b+d-c)(\alpha+\gamma-\beta)(1+a)+4(a \beta(b+d)+c(\alpha+\gamma))>0, \alpha+\gamma>\beta, b+d>c$.

Proof. First suppose that there exists a prime period two solution

$$
\ldots, p, q, p, q, \ldots,
$$

of (1.1). We will prove that condition (i) holds.

We see from (1.1) that

$$
\begin{gathered}
p=a q+\frac{b p+c q+d p}{\alpha p+\beta q+\gamma p}=a q+\frac{e p+c q}{f p+\beta q}, \quad \text { where } e=b+d, f=\alpha+\gamma, \\
q=a p+\frac{b q+c p+d q}{\alpha q+\beta p+\gamma q}=a p+\frac{e q+c p}{f q+\beta p} .
\end{gathered}
$$

Then

$$
\begin{aligned}
& f p^{2}+\beta p q=a f p q+a \beta q^{2}+e p+c q \\
& f q^{2}+\beta p q=a f p q+a \beta p^{2}+e q+c p .
\end{aligned}
$$

Subtracting (4.3) from (4.4) gives

$$
f\left(p^{2}-q^{2}\right)=-a \beta\left(p^{2}-q^{2}\right)+(e-c)(p-q)
$$

Since $p \neq q$, it follows that

$$
p+q=\frac{(e-c)}{(f+a \beta)}
$$

Again, adding (4.3) and (4.4) yields

$$
f\left(p^{2}+q^{2}\right)+2 \beta p q=2 a f p q+a \beta\left(p^{2}+q^{2}\right)+(e+c)(p+q)
$$

or

$$
(f-a \beta)\left(p^{2}+q^{2}\right)+2(\beta-a f) p q=(e+c)(p+q) .
$$


It follows by (4.6), (4.8) and the relation

$$
p^{2}+q^{2}=(p+q)^{2}-2 p q \quad \forall p, q \in R
$$

that

$$
2(\beta-f)(1+a) p q=\frac{2(e a \beta+c f)(e-c)}{(f+a \beta)^{2}} .
$$

Thus

$$
p q=\frac{(e a \beta+c f)(e-c)}{(f+a \beta)^{2}(\beta-f)(1+a)} .
$$

Now it is clear from (4.6) and (4.11) that $p$ and $q$ are the two distinct roots of the quadratic equation

$$
\begin{aligned}
& t^{2}-\left(\frac{(e-c)}{(f+a \beta)}\right) t+\left(\frac{(e a \beta+c f)(e-c)}{(f+a \beta)^{2}(\beta-f)(1+a)}\right)=0 \\
& (f+a \beta) t^{2}-(e-c) t+\left(\frac{(e a \beta+c f)(e-c)}{(f+a \beta)(\beta-f)(1+a)}\right)=0
\end{aligned}
$$

and so

$$
[e-c]^{2}-\frac{4(e a \beta+c f)(e-c)}{(\beta-f)(1+a)}>0
$$

or

$$
\begin{gathered}
{[e-c]^{2}+\frac{4(e a \beta+c f)(e-c)}{(f-\beta)(1+a)}>0,} \\
(e-c)(f-\beta)(1+a)+4(e a \beta+c f)>0 .
\end{gathered}
$$

Therefore, inequality (i) holds.

Second, suppose that inequality (i) is true. We will show that (1.1) has a prime period two solution.

Assume that

$$
\begin{aligned}
& p=\frac{e-c+\zeta}{2(f+a \beta)}, \\
& q=\frac{e-c-\zeta}{2(f+a \beta)},
\end{aligned}
$$

where $\zeta=\sqrt{[e-c]^{2}-4(e a \beta+c f)(e-c) /(\beta-f)(1+a)}$. 
Discrete Dynamics in Nature and Society

We see from inequality (i) that

$$
(e-c)(f-\beta)(1+a)+4(e a \beta+c f)>0, \quad e>c, f>\beta,
$$

which is equivalent to

$$
(e-c)^{2}>\frac{4(e a \beta+c f)(e-c)}{(\beta-f)(1+a)} .
$$

Therefore, $p$ and $q$ are distinct real numbers.

Set

$$
x_{-3}=p, \quad x_{-2}=q, \quad x_{-1}=p, \quad x_{0}=q .
$$

We wish to show that

$$
x_{1}=x_{-1}=p, \quad x_{2}=x_{0}=q .
$$

It follows from (1.1) that

$$
\begin{aligned}
x_{1} & =a q+\frac{b p+c q+d p}{\alpha p+\beta q+\gamma p}=a q+\frac{e p+c q}{f p+\beta q} \\
& =a\left(\frac{e-c-\zeta}{2(f+a \beta)}\right)+\frac{e((e-c+\zeta) / 2(f+a \beta))+c((e-c-\zeta) / 2(f+a \beta))}{f((e-c+\zeta) / 2(f+a \beta))+\beta((e-c-\zeta) / 2(f+a \beta))} .
\end{aligned}
$$

Dividing the denominator and numerator by $2(f+a \beta)$ gives

$$
\begin{aligned}
x_{1} & =\frac{a e-a c-a \zeta}{2(f+a \beta)}+\frac{e(e-c+\zeta)+c(e-c-\zeta)}{f(e-c+\zeta)+\beta(e-c-\zeta)} \\
& =\frac{a e-a c-a \zeta}{2(f+a \beta)}+\frac{(e-c)[(e+c)+\zeta]}{(f+\beta)(e-c)+(f-\beta) \zeta} .
\end{aligned}
$$


Multiplying the denominator and numerator of the right side by $(f+\beta)(e-c)-(f-\beta) \zeta$ gives

$$
\begin{aligned}
x_{1}= & \frac{a e-a c-a \zeta}{2(f+a \beta)}+\frac{(e-c)[(e+c)+\zeta][(f+\beta)(e-c)-(f-\beta) \zeta]}{[(f+\beta)(e-c)+(f-\beta) \zeta][(f+\beta)(e-c)-(f-\beta) \zeta]} \\
= & \frac{a e-a c-a \zeta}{2(f+a \beta)} \\
& +\frac{(e-c)\left\{(f+\beta)\left(e^{2}-c^{2}\right)+\zeta[(f+\beta)(e-c)-(f-\beta)(e+c)]-(f-\beta) \zeta^{2}\right\}}{(f+\beta)^{2}(e-c)^{2}-(f-\beta)^{2} \zeta^{2}} \\
= & \frac{a e-a c-a \zeta}{2(f+a \beta)}+\frac{(e-c)\left\{(f+\beta)\left(e^{2}-c^{2}\right)+2 \zeta(\beta e-c f)-(f-\beta) A\right\}}{(f+\beta)^{2}(e-c)^{2}-(f-\beta)^{2} A} \\
= & \frac{a e-a c-a \zeta}{2(f+a \beta)}+\frac{(e-c)\left\{(f+\beta)\left(e^{2}-c^{2}\right)+2 \zeta(\beta e-c f)-B\right\}}{(f+\beta)^{2}(e-c)^{2}-(f-\beta)^{2} A} \\
= & \frac{a e-a c-a \zeta}{2(f+a \beta)}+\frac{(e-c)\{2(e-c)[f c+\beta e-2(e a \beta+c f) /(1+a)]+2 \zeta(\beta e-c f)\}}{4(e-c)[\beta f(e-c)+(\beta-f)(e a \beta+c f) /(1+a)]},
\end{aligned}
$$

where $\mathcal{A}$ denotes to $[e-c]^{2}-4(e a \beta+c f)(e-c) /(\beta-f)(1+a)$ and $B$ denotes to $(f-\beta)(e-c)^{2}+$ $4(e a \beta+c f)(e-c) /(1+a)$.

Multiplying the denominator and numerator of the right side by $(1+a)$, we obtain

$$
\begin{aligned}
x_{1} & =\frac{a e-a c-a \zeta}{2(f+a \beta)}+\frac{(e-c)[(f c+\beta e)(1+a)-2(e a \beta+c f)]+\zeta(1+a)(\beta e-c f)}{2[\beta f(e-c)(1+a)+(\beta-f)(e a \beta+c f)]} \\
& =\frac{a e-a c-a \zeta}{2(f+a \beta)}+\frac{(e-c)(\beta e-f c)(1-a)+\zeta(1+a)(\beta e-c f)}{2[\beta f(e-c)(1+a)+(\beta-f)(e a \beta+c f)]} \\
& =\frac{a e-a c-a \zeta}{2(f+a \beta)}+\frac{(\beta e-f c)\{(e-c)(1-a)+\zeta(1+a)\}}{2(\beta e-c f)(f+a \beta)} \\
& =\frac{a e-a c-a \zeta}{2(f+a \beta)}+\frac{(e-c)(1-a)+\zeta(1+a)}{2(f+a \beta)} \\
& =\frac{a e-a c-a \zeta+(e-c)(1-a)+\zeta(1+a)}{2(f+a \beta)}=\frac{e-c+\zeta}{2(f+a \beta)}=p .
\end{aligned}
$$

Similarly as before one can easily show that

$$
x_{2}=q \text {. }
$$


Then it follows by induction that

$$
x_{2 n}=q, \quad x_{2 n+1}=p \quad \forall n \geq-1 .
$$

Thus (1.1) has the prime period two solution

$$
\ldots, p, q, p, q, \ldots,
$$

where $p$ and $q$ are the distinct roots of the quadratic equation (4.13) and the proof is complete.

\section{Global Attractor of the Equilibrium Point of (1.1)}

In this section, we investigate the global asymptotic stability of (1.1).

Lemma 5.1. For any values of the quotient $b / \alpha, c / \beta$, and $d / \gamma$, the function $f(u, v, w, t)$ defined by (2.3) has the monotonicity behavior in its three arguments.

Proof. The proof follows by some computations and it will be omitted.

Remark 5.2. It follows from (1.1), when $b / \alpha=c / \beta=d / \gamma$, that

$$
x_{n+1}=a x_{n}+\lambda \quad \forall n \geq-3 \text { and for some constant } \lambda .
$$

Whenever the quotients $\alpha / A, \beta / B$, and $\gamma / C$ are not equal, we get the following result.

Theorem 5.3. The equilibrium point $\bar{x}$ is a global attractor of (1.1) if one of the following statements holds:
(1) $\frac{b}{\alpha} \geq \frac{c}{\beta} \geq \frac{d}{\gamma}, \quad d \geq b+c$,
(2) $\frac{b}{\alpha} \geq \frac{d}{\gamma} \geq \frac{c}{\beta}, \quad c \geq b+d$,
(3) $\frac{c}{\beta} \geq \frac{b}{\alpha} \geq \frac{d}{\gamma}, \quad d \geq b+c$,
(4) $\frac{c}{\beta} \geq \frac{d}{\gamma} \geq \frac{b}{\alpha}, \quad b \geq c+d$,
(5) $\frac{d}{\gamma} \geq \frac{c}{\beta} \geq \frac{b}{\alpha}, \quad b \geq c+d$,
(6) $\frac{d}{\gamma} \geq \frac{b}{\alpha} \geq \frac{c}{\beta}, \quad c \geq b+d$. 
Proof. Let $\left\{x_{n}\right\}_{n=-3}^{\infty}$ be a solution of (1.1) and again let $f$ be a function defined by (2.3).

We will prove the theorem when case (1.1) is true, and the proof of the other cases is similar and so will be omitted.

Assume that (5.2) is true, then it is easy from the equations after (2.3) to see that the function $f(u, v, w, t)$ is nondecreasing in $u, v$ and nonincreasing in $t$ and it is not clear what is going on with $w$. So we consider the following two cases.

Case 1. Assume that the function $f(u, v, w, t)$ is nondecreasing in $w$.

Suppose that $(m, M)$ is a solution of the system $M=f(M, M, M, m)$ and $m=$ $g(m, m, m, M)$. Then from (1.1), we see that

$$
M=a M+\frac{b M+c M+d m}{\alpha M+\beta M+\gamma m}, \quad m=a m+\frac{b m+c m+d M}{\alpha m+\beta m+\gamma M},
$$

or

$$
M(1-a)=\frac{b M+c M+d m}{\alpha M+\beta M+\gamma m}, \quad m(1-a)=\frac{b m+c m+d M}{\alpha m+\beta m+\gamma M} .
$$

Then

$$
\begin{gathered}
(\alpha+\beta)(1-a) M^{2}+\gamma(1-a) M m=(b+c) M+d m, \\
(\alpha+\beta)(1-a) m^{2}+\gamma(1-a) M m=(b+c) m+d M .
\end{gathered}
$$

Subtracting this two equations, we obtain

$$
(M-m)\{(\alpha+\beta)(1-a)(M+m)+(d-b-c)\}=0,
$$

under the conditions $d \geq b+c, a<1$, we see that

$$
M=m .
$$

It follows by Theorem B that $\bar{x}$ is a global attractor of (1.1) and then the proof is complete.

Case 2. Assumes that the function $f(u, v, w, t)$ is nonincreasing in $w$.

Suppose that $(m, M)$ is a solution of the system $M=f(M, M, m, m)$ and $m=$ $g(m, m, M, M)$. Then from (1.1), we see that

$$
M=a M+\frac{b M+c M+d m}{\alpha M+\beta M+\gamma m}, \quad m=a m+\frac{b m+c m+d M}{\alpha m+\beta m+\gamma M},
$$

or

$$
M(1-a)=\frac{b M+c m+d m}{\alpha M+\beta m+\gamma m}, \quad m(1-a)=\frac{b m+c M+d M}{\alpha m+\beta M+\gamma M} .
$$




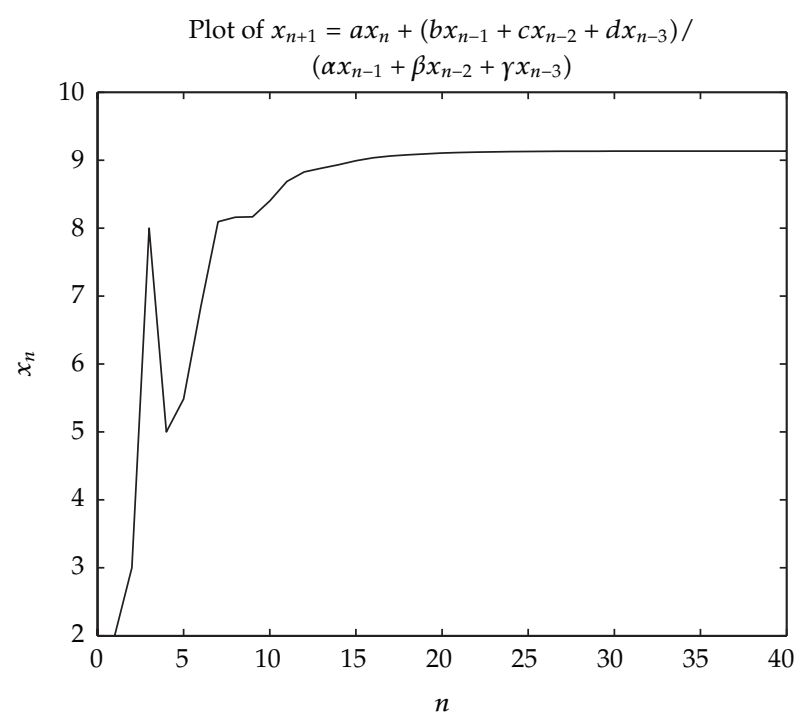

Figure 1

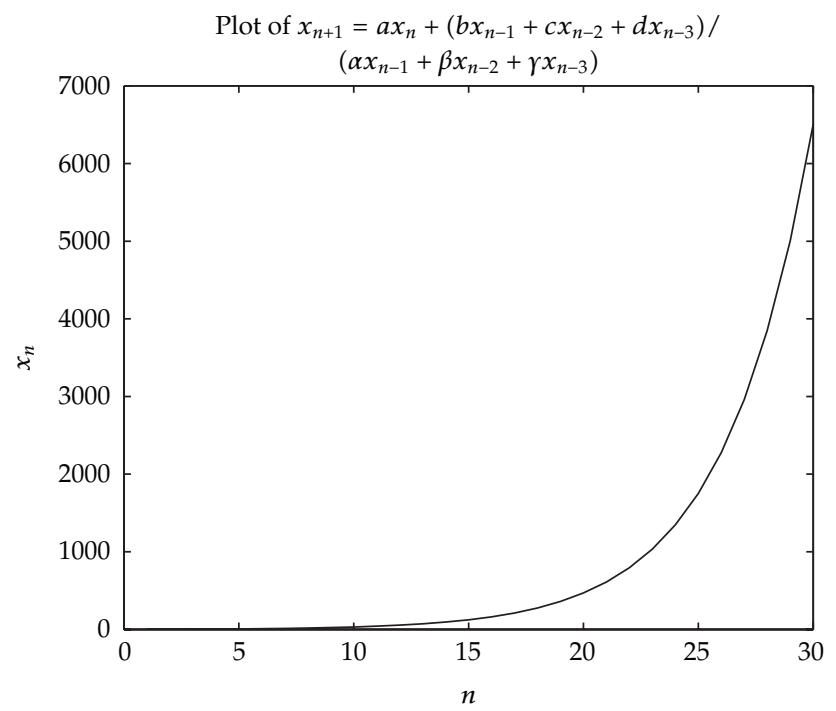

Figure 2

Then

$$
\begin{gathered}
\alpha(1-a) M^{2}+(\beta+\gamma)(1-a) M m=b M+(c+d) m, \\
\alpha(1-a) m^{2}+(\beta+\gamma)(1-a) M m=b m+(c+d) M .
\end{gathered}
$$

Subtracting these two equations we obtain

$$
(M-m)\{\alpha(1-a)(M+m)+(c+d-b)\}=0 .
$$




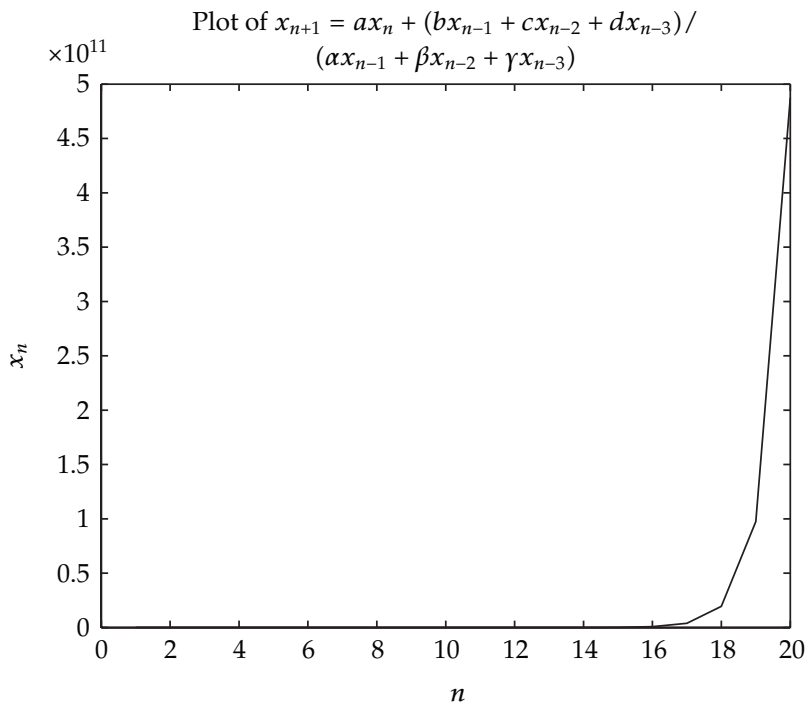

Figure 3

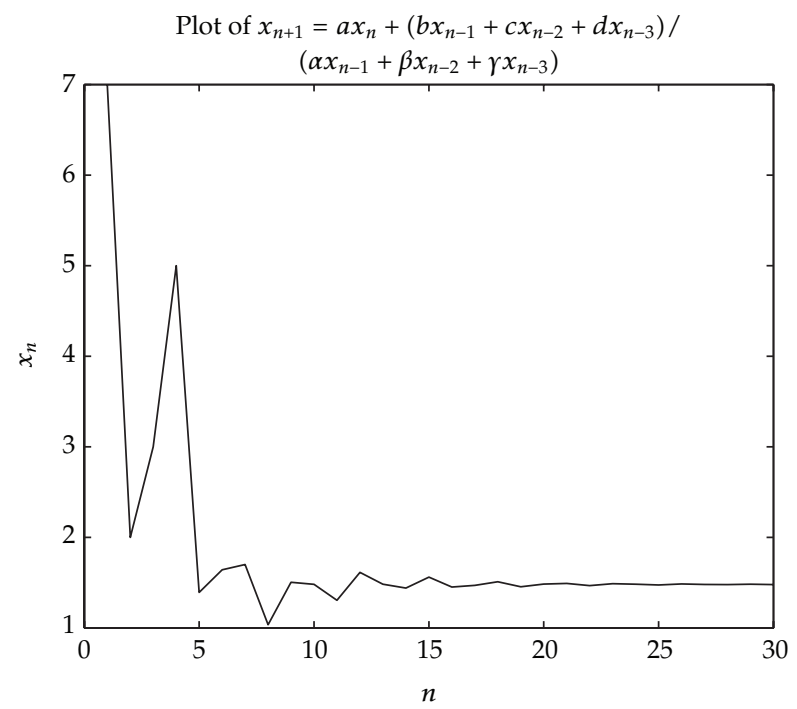

Figure 4

Under the conditions $d \geq b+c, a<1$, we see that

$$
M=m .
$$

It follows by Theorem B that $\bar{x}$ is a global attractor of (1.1) and then the proof is complete. 


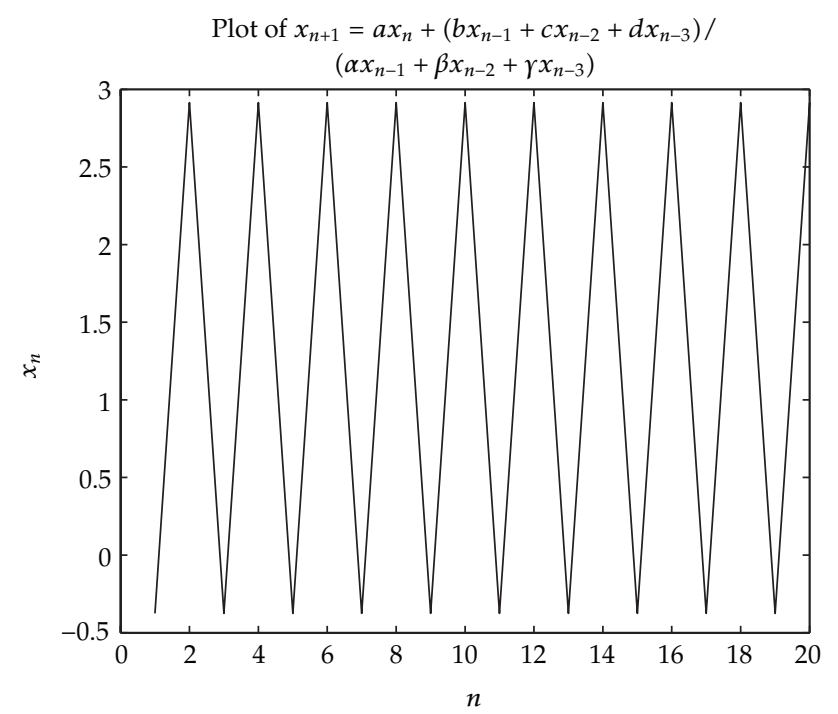

Figure 5

\section{Numerical Examples}

For confirming the results of this paper, we consider numerical examples which represent different types of solutions to (1.1).

Example 6.1. We assume $x_{-3}=2, x_{-2}=3, x_{-1}=8, x_{0}=5, a=0.6, b=7, c=3, d=9, \alpha=$ 3.8, $\beta=0.2$, and $\gamma=1.2$. See Figure 1 .

Example 6.2 (see [Figure 2]). Since $x_{-3}=5, x_{-2}=6, x_{-1}=3, x_{0}=4, a=1.3, b=5, c=$ $3, d=8, \alpha=6, \beta=4$, and $\gamma=7$.

Example 6.3. We consider $x_{-3}=5, x_{-2}=7, x_{-1}=11, x_{0}=3, a=5, b=2, c=3, d=4, \alpha=$ $3, \beta=2$, and $\gamma=7$. See Figure 3 .

Example 6.4 (see [Figure 4]). Since $x_{-3}=7, x_{-2}=2, x_{-1}=3, x_{0}=5, a=0.1, b=2, c=$ $5, d=1.4, \alpha=1.3, \beta=2$, and $\gamma=3$.

Example 6.5 (see [Figure 5]). It shows the solutions when $x_{-3}=x_{-1}=p, x_{-2}=x_{0}=q, a=$ $0.6, b=7, c=3, d=9, \alpha=3.8, \beta=0.2, \gamma=1.2$

$$
\text { Since } p, q=\left(\frac{(e-c) \pm \sqrt{[e-c]^{2}-4(e a \beta+c f)(e-c) /(\beta-f)(1+a)}}{2(f+a \beta)}\right) \text {. }
$$

\section{References}

[1] R. P. Agarwal and E. M. Elsayed, "On the solution of fourth-order rational recursive sequence," Advanced Studies in Contemporary Mathematics, vol. 20, no. 4, pp. 525-545, 2010.

[2] E. M. Elabbasy, H. El-Metwally, and E. M. Elsayed, "Some properties and expressions of solutions for a class of nonlinear difference equation," Utilitas Mathematica, vol. 87, pp. 93-110, 2012. 
[3] E. M. Elabbasy, H. El-Metwally, and E. M. Elsayed, "On the difference equation $x_{n+1}=a x_{n}-$ $\left(b x_{n}\right) /\left(c x_{n}-d x_{n-1}\right), "$ Advances in Difference Equations, vol. 2006, Article ID 82579, 10 pages, 2006.

[4] E. M. Elabbasy, H. El-Metwally, and E. M. Elsayed, "Global behavior of the solutions of difference equation," Advances in Difference Equations, vol. 2011, article 28, 2011.

[5] E. M. Elabbasy and E. M. Elsayed, "On the global attractivity of difference equation of higher order," Carpathian Journal of Mathematics, vol. 24, no. 2, pp. 45-53, 2008.

[6] E. M. Elabbasy and E. M. Elsayed, "Global attractivity and periodic nature of a difference equation," World Applied Sciences Journal, vol. 12, no. 1, pp. 39-47, 2011.

[7] E. M. Elsayed, "On the solution of some difference equations," European Journal of Pure and Applied Mathematics, vol. 4, no. 3, pp. 287-303, 2011.

[8] E. M. Elsayed, "Dynamics of a recursive sequence of higher order," Communications on Applied Nonlinear Analysis, vol. 16, no. 2, pp. 37-50, 2009.

[9] E. M. Elsayed, "Solution and attractivity for a rational recursive sequence," Discrete Dynamics in Nature and Society, vol. 2011, Article ID 982309, 17 pages, 2011.

[10] E. M. M. Elsayed, "Behavior of a rational recursive sequences," Studia. Universitatis Babeş-Bolyai Mathematica, vol. 56, no. 1, pp. 27-42, 2011.

[11] E. M. Elsayed, "On the global attractivity and the solution of recursive sequence," Studia Scientiarum Mathematicarum Hungarica, vol. 47, no. 3, pp. 401-418, 2010.

[12] E. M. Elsayed, "On the dynamics of a higher-order rational recursive sequence," Communications in Mathematical Analysis, vol. 12, no. 1, pp. 117-133, 2012.

[13] E. M. Elsayed, "Dynamics of recursive sequence of order two," Kyungpook Mathematical Journal, vol. 50, no. 4, pp. 483-497, 2010.

[14] E. M. Elsayed, "Solutions of rational difference systems of order two," Mathematical and Computer Modelling, vol. 55, no. 3-4, pp. 378-384, 2012.

[15] E. A. Grove and G. Ladas, Periodicities in Nonlinear Difference Equations, vol. 4, Chapman \& Hall/CRC, Boca Raton, Fla, USA, 2005.

[16] M. Saleh and M. Aloqeili, "On the rational difference equation $x_{n+1}=A+x_{n} / x_{n-K}$," Applied Mathematics and Computation, vol. 171, no. 2, pp. 862-869, 2005.

[17] X. Yang, W. Su, B. Chen, G. M. Megson, and D. J. Evans, "On the recursive sequence $x_{n+1}=\left(a x_{n-1}+\right.$ $\left.b x_{n-2}\right) /\left(c+d x_{n-1} x_{n-2}\right)$," Applied Mathematics and Computation, vol. 162, no. 3, pp. 1485-1497, 2005.

[18] V. L. Kocić and G. Ladas, Global Behavior of Nonlinear Difference Equations of Higher Order with Applications, vol. 256, Kluwer Academic Publishers Group, Dordrecht, The Netherlands, 1993.

[19] M. R. S. Kulenović and G. Ladas, Dynamics of Second Order Rational Difference Equations With Open Problems and Conjectures, Chapman \& Hall/CRC, Boca Raton, Fla, USA, 2002.

[20] T. Sun and H. Xi, "On convergence of the solutions of the difference equation $x_{n+1}=1+x_{n-1} / x_{n}$," Journal of Mathematical Analysis and Applications, vol. 325, no. 2, pp. 1491-1494, 2007.

[21] N. Touafek and E. M. Elsayed, "On the solutions of systems of rational difference equations," Mathematical and Computer Modelling, vol. 55, no. 7-8, pp. 1987-1997, 2012.

[22] I. Yalçinkaya, "On the difference equation $x_{n+1}=\alpha+x_{n-m} / x_{n}^{k}$," Discrete Dynamics in Nature and Society. An International Multidisciplinary Research and Review Journal, Article ID 805460, 8 pages, 2008.

[23] X. Yang, X. Liu, and L. Wang, "Stability of a generalized Putnam equation," Applied Mathematics Letters, vol. 22, no. 4, pp. 565-568, 2009.

[24] X. Yang, D. J. Evans, and G. M. Megson, "Global asymptotic stability in a class of Putnam-type equations," Nonlinear Analysis A, vol. 64, no. 1, pp. 42-50, 2006.

[25] X. Yang, "Global asymptotic stability in a class of generalized Putnam equations," Journal of Mathematical Analysis and Applications, vol. 322, no. 2, pp. 693-698, 2006.

[26] E. M. E. Zayed and M. A. El-Moneam, "On the rational recursive sequence $x_{n+1}=a x_{n}-\left(b x_{n}\right) /\left(c x_{n}+\right.$ $\left.d x_{n-k}\right)$," Communications on Applied Nonlinear Analysis, vol. 15, no. 2, pp. 47-57, 2008. 


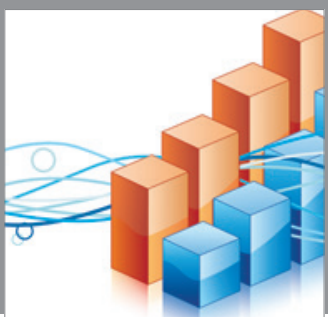

Advances in

Operations Research

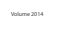

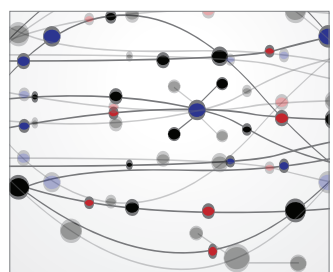

\section{The Scientific} World Journal
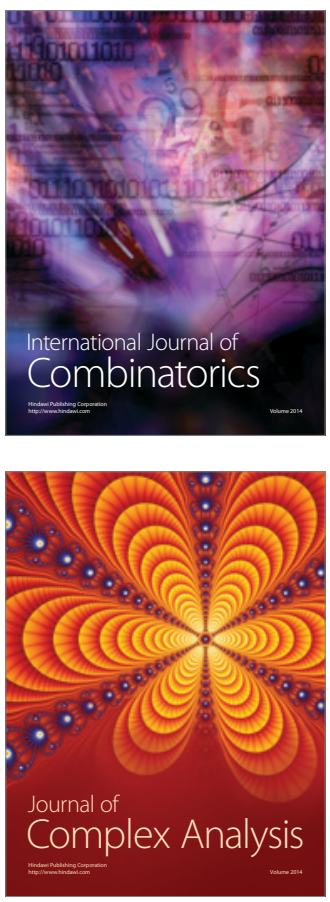

International Journal of

Mathematics and

Mathematical

Sciences
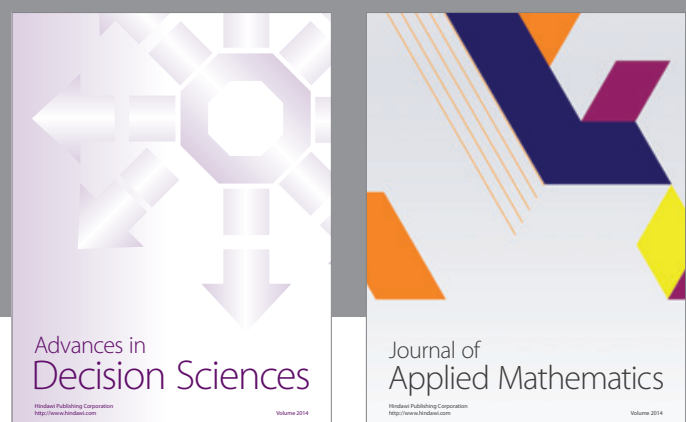

Journal of

Applied Mathematics
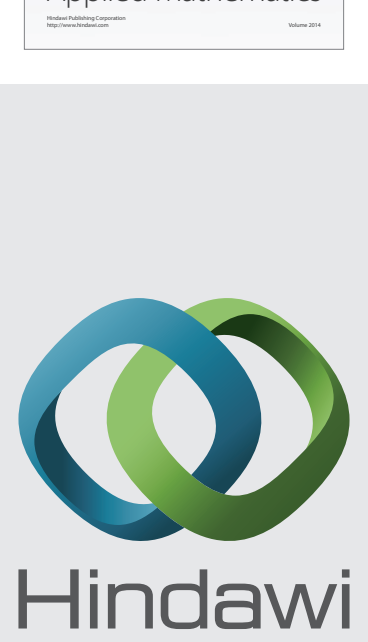

Submit your manuscripts at http://www.hindawi.com
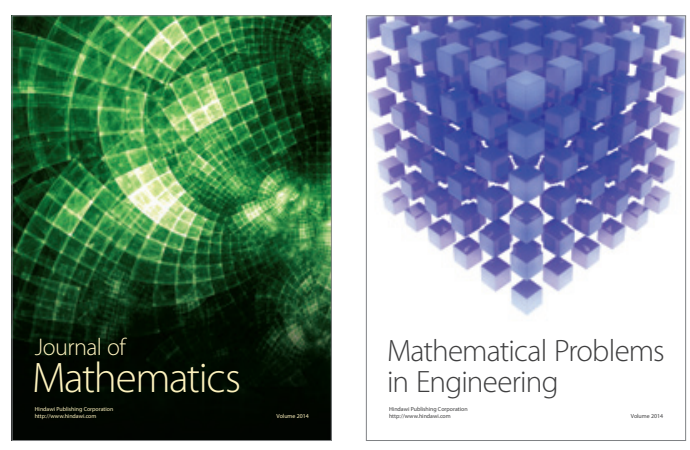

Mathematical Problems in Engineering
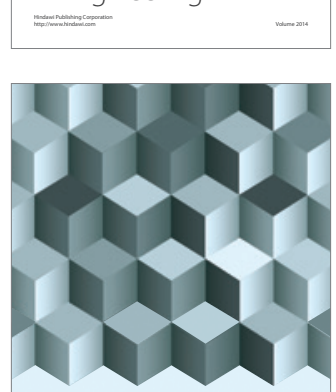

Journal of

Function Spaces
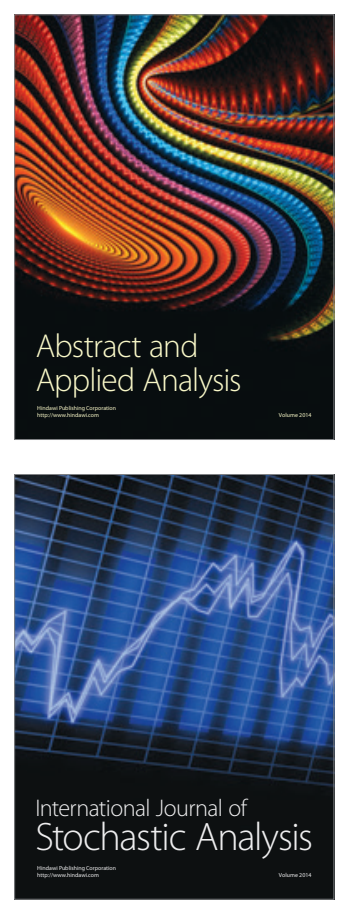

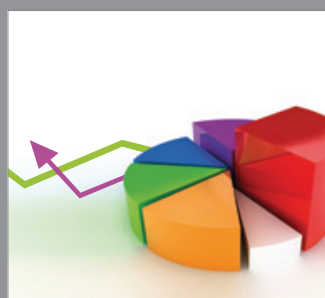

ournal of

Probability and Statistics

Promensencen
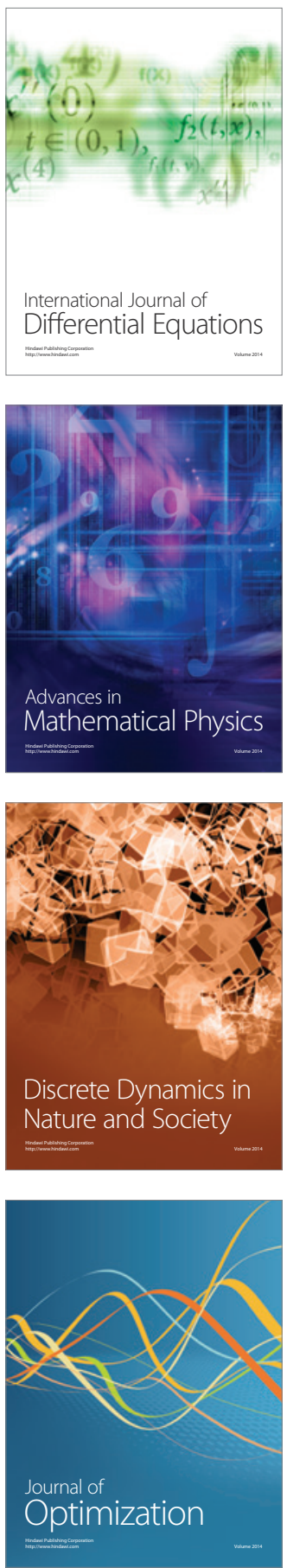